An isolated, implausibly large effect in a case-control study of digit ratio (2D:4D) and male androgenetic alopecia: Comment on Unal (J Cosmet Dermatol 2018;17(3):545-548)

\author{
Martin Voracek, DSc, DMSc, PhD, MSc, MPhil \\ Department of Basic Psychological Research, School of Psychology, \\ University of Vienna, Austria
}

This manuscript version: September 9, 2017

Correspondence: martin.voracek@univie.ac.at 


\section{An isolated, implausibly large effect in a case-control study of digit ratio (2D:4D) and male androgenetic alopecia: Comment on Unal (J Cosmet Dermatol 2018;17(3):545-548)}

Unal reported a case-control study ${ }^{1}$ on the association between digit ratio (2D:4D; a purported retrospective anatomical marker for prenatal androgen action) and male androgenetic alopecia (AGA), concluding that left-hand (L2D:4D), but not right-hand (R2D:4D), digit ratio is a possible indicator of AGA. Key descriptive statistics $(M \pm S D)$ of this study were $0.973 \pm 0.033$ vs. $0.972 \pm 0.035$ for R2D:4D among 99 male cases (with grade III or more on the Hamilton-Norwood scale of hair loss) vs. 90 male controls, but 0.893 [sic] \pm 0.026 (cases) vs. $0.971 \pm 0.028$ (controls) for L2D:4D.

It is easy to see that three of these four group $M$ values virtually are identical, only one deviates (L2D:4D in cases). To what extent, is illustrated by effect-size analysis. Cohen's $d$ (the standardized group mean difference) was 0.029 for the case-control difference in R2D:4D, but 2.892 (about 100 times larger) for the case-control difference in L2D:4D. Likewise, the right-left difference in 2D:4D was $d=0.032$ among controls, but $d=2.693$ (about 85 times larger) among cases.

To put these figures in further perspective, $d$ values around $0.20,0.50$, and 0.80 represent widely used benchmarks standing for small, medium, and large effects, respectively. As for a readily understood everyday-life example for what is a very large effect, male-female differences observed for human height (among adults of same age and region) often amount to $d$ $\approx 1.70$ (given that men, on average, are about $12 \mathrm{~cm}$ taller than women, and within-sex standard deviations of height are about $7 \mathrm{~cm}$ among both sexes). Even so, the effect size of right-left differences in cases' $2 \mathrm{D}: 4 \mathrm{D}(d=2.693)$ in the Unal study was about $60 \%$ larger than a truly very large effect, such as male-female differences in height.

Such an exceedingly large right-left difference in 2D:4D, notabene observed within the same group of individuals, is unheard-of in the 2D:4D research literature. As well, it does not conform with everyday observations and for this reason alone a priori is implausible. It is 
obvious that, on average, individuals do not present left-hand index fingers of not even $90 \%$ (Unal study: $M=0.893$ ) the length of their left-hand ring fingers, when, on average, their righthand index fingers are almost of equal length (Unal study: $M=0.973$ ) than their right-hand ring fingers. Human hand anatomy, including finger-length patterns, shows much higher degrees of right-left symmetry.

The main finding in the Unal study, namely, an association of L2D:4D (but not R2D:4D) and AGA, is entirely due to an incredibly large right-left difference in 2D:4D in the case group. Such being the case, it follows that the study's main finding itself is not trustworthy. Specifically, the finding would entail that AGA could almost perfectly be predicted from L2D:4D, whereas not at all from R2D:4D. A further, possibly related, concern is that Unal claimed that the fingerlength measurements (for calculating 2D:4D) were done blinded to group status (case vs. control individuals). However, this is impossible, because fingers were measured directly with a caliper, i.e., face-to-face to study participants, and AGA is seeable. In addition, cases on average were 10 yr. older than controls.

Effects observed in analogous studies of 2D:4D and male AGA invariably have been small or nil. In another case-control study² (189 cases, 171 controls), effects were $d=-0.386$ (R2D:4D) and -0.161 (L2D:4D). In a much larger ( $n=1200$ men) correlational study33, both R2D:4D and L2D:4D were unrelated ( $r=.025$ and .028$)$ to AGA severity, as assessed with the HamiltonNorwood scale. Finally, in yet another case-control study ${ }^{4}$ (83 cases, 70 controls), effects likewise were nil, $d=-0.099$ (R2D:4D) and -0.022 (L2D:4D), as was the case in a further correlational study $(n=196)^{5}$. These latter two studies were not cited by Unal.

In conclusion, the cumulated research evidence suggests 2D:4D associations with AGA either are negligible or nonexistent. 2D:4D most likely is not an indicator of male AGA. The magnitude of the isolated L2D:4D effect, as reported in the Unal study, deviates so conspicuously from all analogous studies as to raise the question of whether it is veridical. 


\section{References}

1. Unal M. Digit ratio 2D:4D is a possible indicator for androgenetic alopecia in males. J Cosmet Dermatol. 2017, doi: 10.1111/jocd.12403.

2. Bilgiç Ö, Altınyazar HC, Eryılmaz D, Tuğrul ZA. Are 2D:4D finger-length ratios an indicator of androgenetic alopecia in males? An Bras Dermatol. 2016;91:156-159.

3. Feily A, Hosseinpoor M, Bakhti A, et al. Digit-length ratios (2D:4D) as a phenotypic indicator of in utero androgen exposure is not prognostic for androgenic alopecia: A descriptive-analytic study of 1200 Iranian men. Dermatol Reports. 2016;8:6386.

4. Birch P, Messenger A. Finger length ratios in male androgenetic alopecia: Is there an inutero effect of androgens? J Invest Dermatol. 2006;126(S1):104.

5. Margel D, Venkateswaran S, Darwish A, et al. The association between male pattern baldness and second to fourth finger ratio with prostate cancer: A prospective cohort study. J Urol. 2012;187(S4):e588. 